\title{
Fatty acid desaturases in human adipose tissue: relationships between gene expression, desaturation indexes and insulin resistance
}

\author{
P. Sjögren • J. Sierra-Johnson • K. Gertow $•$ M. Rosell • \\ B. Vessby • U. de Faire • A. Hamsten • M.-L. Hellenius • \\ R. M. Fisher
}

Received: 28 June 2007 / Accepted: 15 October 2007 / Published online: 20 November 2007

(C) Springer-Verlag 2007

\begin{abstract}
Aims/hypothesis Fatty acid desaturases introduce double bonds into growing fatty acid chains. The key desaturases in humans are $\Delta^{5}$-desaturase (D5D), $\Delta^{6}$-desaturase (D6D) and stearoyl-CoA desaturase (SCD). Animal and human data implicate hepatic desaturase activities in insulin resistance, obesity and dyslipidaemia. However, the role of desaturase activity in adipose tissue is uncertain. We therefore evaluated relationships between adipose mRNA expression, estimated desaturase activities (fatty acid ratios) in adipose tissue and insulin resistance.

Methods Subcutaneous adipose tissue mRNA expression of $D 5 D$ (also known as FADS1), D6D (also known as FADS2) and $S C D$ was determined in 75 individuals representative of the study population of 294 healthy 63-year-old men.
\end{abstract}

P. Sjögren · J. Sierra-Johnson · K. Gertow · A. Hamsten •

M.-L. Hellenius $\cdot$ R. M. Fisher $(\bowtie)$

Atherosclerosis Research Unit, Department of Medicine (Solna),

Karolinska Institutet, Karolinska University Hospital,

17176 Stockholm, Sweden

e-mail: rachel.fisher@ki.se

J. Sierra-Johnson

Department of Internal Medicine,

Division of Cardiovascular Diseases, Mayo Clinic,

Rochester, MN, USA

M. Rosell • U. de Faire

Division of Cardiovascular Epidemiology,

Institute of Environmental Medicine, Karolinska Institutet,

Stockholm, Sweden

B. Vessby

Department of Public Health and Caring Sciences,

University of Uppsala,

Uppsala, Sweden
Desaturation indexes (product/substrate fatty acid ratios) were generated from adipose tissue fatty acid composition in all individuals. Insulin resistance was defined as the upper quartile of the updated homeostasis model assessment (HOMA-2) index.

Results The relevant desaturation indexes (16:1/16:0, 18:1/18:0, 20:4/20:3 and 18:3/18:2) reflected expression of $S C D$, but not of $D 5 D$ or $D 6 D$ in adipose tissue. Insulin-resistant individuals had a higher adipose tissue 18:1/18:0, but not 16:1/16:0 ratio than insulin-sensitive individuals. Individuals with a high adipose tissue 18:1/18:0 ratio were 4.4-fold $(95 \%$ CI 1.8-11.8) more likely to be insulin resistant [threefold (95\% CI 1.1-8.6) after adjustment for waist circumference and plasma triacylglycerol]. In a multiple regression model predicting HOMA-2, the independent effect of the 18:1/18:0 ratio was borderline $(p=0.086)$.

Conclusions/interpretation Adipose tissue desaturation indexes of SCD reflect the expression of the gene encoding the enzyme in this tissue. Elevated SCD activity within adipose tissue is closely coupled to the development of insulin resistance.

Keywords Adipose tissue - Desaturase .

Fatty acid composition · Human · Insulin resistance ·

Stearoyl-CoA desaturase

$\begin{array}{ll}\text { Abbreviations } \\ \text { CRP } & \text { C-reactive protein } \\ \text { D5D } & \Delta^{5} \text {-desaturase } \\ \text { D6D } & \Delta^{6} \text {-desaturase } \\ \text { HOMA-2 } & \begin{array}{l}\text { updated homeostasis model assessment } \\ \text { (for insulin sensitivity) }\end{array} \\ \text { SCD } & \text { stearoyl-CoA desaturase }\end{array}$




\section{Introduction}

Insulin resistance and metabolic disturbances are increasing continuously throughout the world, with a correspondingly discouraging future prognosis for type 2 diabetes and cardiovascular disease [1]. To counter this development, it is necessary to understand the complex aetiology underlying these traits.

One component implicated in the insulin-resistant state is a disturbed fatty acid metabolism. Desaturases are key enzymes in the remodelling of fatty acids, introducing a double-bond at the $\Delta^{5}, \Delta^{6}$ or $\Delta^{9}$ carbon of the growing fatty acid chain (Fig. 1). These desaturases are consequently termed $\Delta^{5}$-desaturase (D5D), $\Delta^{6}$-desaturase (D6D) and $\Delta^{9}$-desaturase. Stearoyl-CoA desaturase (SCD) is the key $\Delta^{9}$-desaturase in man. The activities of these enzymes produce fatty acids with a greater degree of desaturation, which are required for the efficient synthesis of more complex lipids, such as triacylglycerols [2]. Both animal and human studies suggest that fatty acid desaturases play a role in various metabolic disturbances, such as insulin resistance and dyslipidaemia [3-5].

Special attention has been paid to SCD, which is the main enzyme responsible for converting saturated fatty acids into monounsaturated fatty acids (16:0 to $16: 1$ and 18:0 to $18: 1$ ). The potential importance of SCD activity has been extensively studied in mouse models, indicating a considerable impact of SCD on dyslipidaemia and obesity [4]. SCD knockout mice display markedly reduced triacylglycerol synthesis with subsequent reductions in plasma triacylglycerol concentrations, effects that have been attributed to the lack of SCD in the liver of these mice. In addition, SCD knockout mice are resistant to diet-induced obesity [6], possibly due to the absence of SCD activity in adipose tissue. However, the literature contains only very limited data on the importance of desaturase activities in adipose tissue.

Studies on desaturases in humans are few and predominantly based on estimates of desaturation activities derived from the circulating fatty acid composition of fasting blood samples. In the fasting state, most lipids in the circulation are derived from the liver. Therefore, calculating the product/ substrate ratio of fatty acids in circulating lipids generates desaturation indexes that are believed to predominantly reflect hepatic desaturase activity. In line with the findings in SCD knockout mice, a couple of studies have suggested a major lipidaemic effect of SCD also in man [7, 8]. Furthermore, the characteristic fatty acid composition of circulating lipids in insulin-resistant individuals indicates that they have increased SCD and D6D activities and decreased D5D activity [5]. The concept that desaturase activities might affect insulin resistance is supported by a recent study in which estimates of hepatic desaturase activity predicted development of the metabolic syndrome [9]. Importantly, however, these studies have not addressed the role of adipose desaturase activities, an issue that is currently unresolved. Finally, the question of whether adipose tissue desaturation activity can be accurately estimated by desaturation indexes (generated from fatty acid product/substrate ratios) is presently unclear.

Against this background, we determined the expression of $D 5 D$ (also known as FADS1), D6D (also known as FADS2) and $S C D$ mRNAs in adipose tissue of healthy 63-year-old Swedish men with a range of insulin sensitivities and from
Fig. 1 Schematic representation of the major steps in fatty acid remodelling and the roles of desaturases. Vertical pathways represent the different fatty acid families $n-7, n-9, n-6$ and $n-3$. EPA, eicosapentaenoic acid; DHA, docosahexaenoic acid

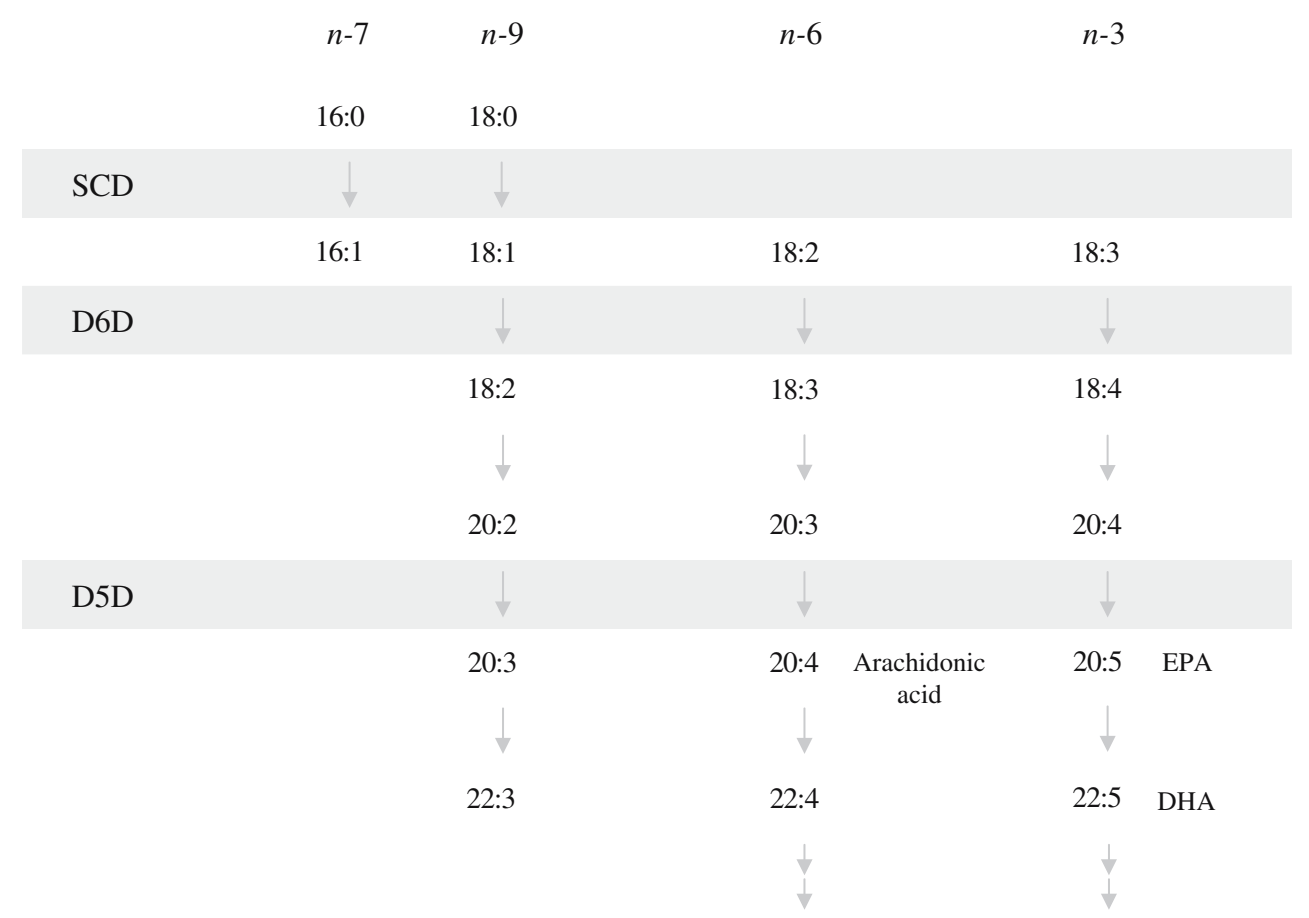


whom adipose tissue fatty acid composition and detailed metabolic profiles were available. Estimates of adipose tissue desaturase activity (fatty acid ratios) and their relationships to desaturase gene expression and insulin resistance were investigated.

\section{Methods}

Participants A biobank and data set comprising a total of 294 healthy, 62- to 64-year-old men (mean $63 \pm 0.6$ years), randomly recruited from a larger population-based cohort of 2039 men living in Stockholm county, was used for this study [10]. The men were selected to represent a wide range of fasting plasma insulin concentrations. Recruitment details, exclusion criteria, clinical procedures and dietary assessment (7 day dietary records) have been described previously [11]. Subcutaneous adipose tissue biopsies were taken from the left upper buttock for subsequent analysis; smaller biopsies were taken from all individuals for fatty acid composition analysis and larger biopsies were taken from a subset of 87 individuals (equally distributed throughout the tertiles of fasting insulin concentrations). Adequate amounts of RNA for expression analysis were obtained from 75 individuals. The Ethics Committee of Karolinska Institutet approved the study and all participants gave informed consent.

Laboratory procedures Methods for determination of plasma glucose, insulin, proinsulin, uric acid, triacylglycerol, LDL peak particle size distribution and cholesterol in VLDL, LDL and HDL have been described previously [11]. Circulating levels of oxidised LDL, C-reactive protein (CRP), TNF $\alpha$ and IL-6 were derived as described previously [12]. RNA was extracted from the subset of larger adipose tissue biopsies and cDNA synthesised as described previously [13]. The expression of $S C D, D 5 D$ and $D 6 D$ mRNAs was quantified in the 75 samples by real-time PCR using an ABI 7000 SDS (Applied Biosystems, Foster City, CA, USA) and normalised for expression of the housekeeping gene RPLPO. Assay numbers for the respective genes were: $S C D$, Hs00748952_s1; $D 5 D$, Hs00203685_m1; D6D, Hs00188654_m1; and for the housekeeping gene RPLP0, Hs99999902 m1 (Assayson-demand; Applied Biosystems). Expression levels were quantified in arbitrary units using a five-point serially diluted cDNA standard curve. The fatty acid composition of subcutaneous adipose tissue was analysed by gas liquid chromatography as described previously [14]. The relative amount of each fatty acid quantified was expressed as the percentage of the total amount of fatty acids.

Estimation of desaturase activity The product/substrate ratios of individual fatty acids in adipose tissue were calculated to estimate the activities of different desaturases as follows: SCD, 16:1/16:0 and 18:1/18:0; D5D, 20:4/20:3; and D6D, 18:3/18:2 (for D5D and D6D, all fatty acids were $n-6$ ).

Statistical procedures JMP 6.0 software (SAS Institute, Cary, $\mathrm{NC}$, USA) was used for statistical analysis, with significance level set to $p<0.05$. Skewed data were logarithmically transformed, but arithmetic means $( \pm \mathrm{SD})$ are presented for ease of understanding. Spearman rank correlation analysis was used to test for relationships between desaturase mRNA expression levels and desaturation indexes and unpaired $t$ test was used to analyse differences in continuous variables between insulin-sensitive and insulin-resistant individuals. Pearson correlation analysis was used in univariate analysis, with the $\log$ of the calculated updated homeostasis model assessment (HOMA-2) value as the dependent variable. HOMA-2 of insulin sensitivity was derived as described previously [15]. Five individuals were excluded with this approach, due to glucose or insulin values outside the ranges of 3.5-25 mmol/1 and $20-400 \mathrm{pmol} / \mathrm{l}$, respectively [16]. Accordingly, 289 individuals remained for group comparisons. Insulin-resistant individuals were defined as those in the upper quartile of HOMA-2 index $(n=61)$. Furthermore, logistic regression analysis, unadjusted and adjusted for (1) high waist circumference and (2) high waist circumference and high plasma triacylglycerol (according to the cutoffs defined by the National Cholesterol Education Program Adult Treatment Panel III (NCEP/ATPIII) for metabolic syndrome components [17]), was used to determine the association between high adipose tissue 18:1/18:0 fatty acid ratio (i.e. upper vs lower quartile of the ratio) and insulin resistance. To evaluate the additional prediction of the 18:1/18:0 ratio, a single parsimonious model was constructed using forward stepwise selection to identify any significant parameters that made additional contributions to the prediction of $\log$ [HOMA-2]. Selection of predictor variables was done in forward stepwise fashion with strict variable entry and elimination criteria. Consequently, the final parsimonious model included only those measures that made independent contributions to the prediction of $\log$ [HOMA-2] (waist circumference and plasma triacylglycerol) plus the adipose tissue 18:1/18:0 ratio.

\section{Results}

Quantification of adipose tissue mRNA levels in the subset of 75 individuals revealed expression levels of $D 5 D, D 6 D$ and $S C D$ to be correlated (Table 1). In particular, a strong relationship between the mRNA levels of $D 5 D$ and $D 6 D$ was found. To investigate whether the fatty acid composition of lipids in adipose tissue reflected the mRNA levels of the different desaturases, desaturation indexes were created from the fatty acids considered to be the typical products 
Table 1 Relationships between the mRNA expression levels of different desaturases and indexes of desaturase activity in human subcutaneous adipose tissue

\begin{tabular}{llll}
\hline \multicolumn{4}{c}{ Adipose tissue mRNA expression } \\
\cline { 2 - 4 } & $S C D$ & $D 5 D$ & $D 6 D$ \\
\hline$S C D$ & & $0.39^{*}$ & $0.47^{* *}$ \\
$D 5 D$ & & $0.70^{* *}$ \\
Adipose tissue FA ratios & & \\
16:1/16:0 $\Delta 9$ & $0.36^{*}$ & & \\
$18: 1 / 18: 0 \Delta 9$ & $0.35^{*}$ & 0.19 & 0.12 \\
$20: 4 / 20: 3 \Delta 5$ & & & \\
$18: 3 / 18: 2 \Delta 6$ & & & \\
\hline
\end{tabular}

$n=75$

Associations are presented as Spearman rank correlation coefficients Levels of mRNA expression were normalised to RPLPO ${ }^{*} p<0.01 ; * * p<0.0001$

FA, fatty acid

and substrates of the respective enzymes. The adipose tissue fatty acid ratios $16: 1 / 16: 0$ and 18:1/18:0 were both significantly correlated with the expression of $S C D$, but there were no significant relationships between the fatty acid ratios 20:4/20:3 and 18:3/18:2 and the expression of D5D and $D 6 D$, respectively (Table 1 ). The two estimates of SCD activity, 16:1/16:0 and 18:1/18:0, were strongly correlated $(r=0.78, p<0.0001)$.

In the study population as a whole (composed of individuals with a range of fasting plasma insulin concentrations), the HOMA-2 index was calculated to estimate the degree of insulin sensitivity. Participants in the upper quartile of HOMA-2 index were classified as insulin resistant and compared with the remaining individuals, who were considered to be insulin sensitive (Table 2). Marked differences were found between the groups, with insulin-resistant individuals having significantly higher plasma concentrations of insulin, proinsulin, glucose, triacylglycerol, uric acid and CRP as well as higher anthropometric measurements and blood pressure. LDL particle size and HDL-cholesterol were significantly lower in the insulin-resistant group, while no significant differences were found for total cholesterol, LDL-cholesterol, oxidised LDL, IL-6 or TNF $\alpha$. Investigation of estimates of SCD activity in adipose tissue (i.e. fatty acid ratios 16:1/16:0 and 18:1/18:0) revealed 18:1/18:0 to be significantly higher in insulin-resistant individuals, but no difference for 16:1/16:0 was found. Differences in dietary fatty acid intake did not seem be able to explain this discrepancy, since the reported intake of 16:0, 16:1, 18:0, 18:1 and the respective ratios did not differ between the insulinresistant and insulin-sensitive groups (data not shown).
Table 2 Characteristics of participants grouped as insulinsensitive or insulin-resistant

Values are means $\pm \mathrm{SD}$

Insulin-resistant individuals were identified according to upper quartile of HOMA-2 index

The $p$ values were calculated from log-transformed data with unpaired $t$ tests

C, cholesterol; FA, fatty acid; oxLDL, oxidised LDL

\begin{tabular}{|c|c|c|c|}
\hline & Insulin-sensitive $(n=228)$ & Insulin-resistant $(n=61)$ & $p$ value \\
\hline \multicolumn{4}{|l|}{ Clinical characteristics } \\
\hline HOMA-2 index & $0.6 \pm 0.2$ & $1.4 \pm 0.4$ & $<0.0001$ \\
\hline Insulin (pmol/1) & $31 \pm 10$ & $76 \pm 23$ & $<0.0001$ \\
\hline Proinsulin (pmol/1) & $3.1 \pm 1.7$ & $5.9 \pm 4.4$ & $<0.0001$ \\
\hline Glucose $(\mathrm{mmol} / \mathrm{l})$ & $4.9 \pm 0.5$ & $5.2 \pm 0.6$ & $<0.0001$ \\
\hline BMI $\left(\mathrm{kg} / \mathrm{m}^{2}\right)$ & $25 \pm 3$ & $28 \pm 3$ & $<0.0001$ \\
\hline Waist $(\mathrm{cm})$ & $94 \pm 8$ & $103 \pm 7$ & $<0.0001$ \\
\hline Uric acid $(\mu \mathrm{mol} / \mathrm{l})$ & $331 \pm 56$ & $365 \pm 66$ & 0.0001 \\
\hline Systolic BP (mmHg) & $133 \pm 14$ & $142 \pm 19$ & 0.0001 \\
\hline Diastolic BP(mmHg) & $80 \pm 8$ & $85 \pm 9$ & $<0.0001$ \\
\hline \multicolumn{4}{|l|}{ Lipids } \\
\hline Triacylglycerol (mmol/l) & $1.1 \pm 0.5$ & $1.6 \pm 0.7$ & $<0.0001$ \\
\hline Cholesterol $(\mathrm{mmol} / \mathrm{l})$ & $5.9 \pm 1.1$ & $5.8 \pm 1.0$ & 0.65 \\
\hline HDL-C (mmol/l) & $1.7 \pm 0.4$ & $1.5 \pm 0.3$ & $<0.0001$ \\
\hline LDL-C (mmol/l) & $3.7 \pm 1.0$ & $3.7 \pm 0.9$ & 0.95 \\
\hline LDL peak $(\AA)$ & $239 \pm 4$ & $237 \pm 5$ & 0.0004 \\
\hline oxLDL (U/1) & $62 \pm 19$ & $61 \pm 18$ & 0.75 \\
\hline oxLDL/LDL-C & $17 \pm 4$ & $17 \pm 5$ & 0.78 \\
\hline \multicolumn{4}{|l|}{ Markers of inflammation } \\
\hline $\mathrm{TNF} \alpha(\mathrm{ng} / \mathrm{l})$ & $2.2 \pm 1.2$ & $2.1 \pm 0.9$ & 0.51 \\
\hline CRP (mg/l) & $1.8 \pm 2.2$ & $2.6 \pm 3.2$ & 0.0068 \\
\hline IL-6 (ng/l) & $1.4 \pm 1.2$ & $1.7 \pm 2.1$ & 0.088 \\
\hline \multicolumn{4}{|l|}{ Adipose tissue FA ratios } \\
\hline $20: 4 / 20 / 3(\Delta 5)$ & $2.6 \pm 0.7$ & $2.5 \pm 0.6$ & 0.69 \\
\hline $18: 3 / 18: 2(\Delta 6)$ & $0.004 \pm 0.002$ & $0.005 \pm 0.002$ & 0.43 \\
\hline 16:1/16:0 (SCD) & $0.36 \pm 0.09$ & $0.37 \pm 0.12$ & 0.56 \\
\hline 18:1/18:0 (SCD) & $14.3 \pm 3.3$ & $16.2 \pm 4.0$ & 0.0004 \\
\hline
\end{tabular}


Although the desaturation indexes for D5D and D6D did not correlate with the mRNA expression level of the respective enzyme (see above), D5D and D6D desaturation indexes in insulin-sensitive and insulin-resistant individuals are presented in Table 2, but there were no significant differences between the groups.

To investigate predictors of insulin sensitivity, univariate analysis was performed with HOMA-2 as the dependent variable (Table 3). HOMA-2 was positively related to BMI, waist circumference, uric acid, blood pressure, triacylglycerol, CRP and the adipose tissue 18:1/18:0 ratio; it was negatively related to HDL-cholesterol and LDL peak. Since adipose tissue 18:1/18:0, but not 16:1/16:0, was related to insulin resistance, the former desaturation index was used in logistic regression analysis to further investigate the impact of the adipose tissue 18:1/18:0 ratio on insulin resistance (Fig. 2). Individuals with a high adipose tissue 18:1/18:0 ratio were 4.4 times (95\% CI 1.8-11.8) more likely to be insulin resistant. This relationship remained significant after adjusting for the two strongest predictors of HOMA-2, waist circumference and plasma triacylglycerol (odds ratio 3.0, 95\% CI 1.1-8.6). The adipose tissue 18:1/18:0 ratio correlated with both BMI and waist circumference $(r=0.34, p<0.0001$ for both), but replacing waist circumference with BMI in the model did not alter the relationships between 18:1/18:0 and insulin resistance (data not shown). In a final multiple regression model predicting the quantitative level of HOMA-2, which included the strongest predictors of HOMA-2 (waist circumference and plasma triacylglycerol), the independent effect of the 18:1/18:0 ratio was borderline ( $p=0.086$; Table 3).

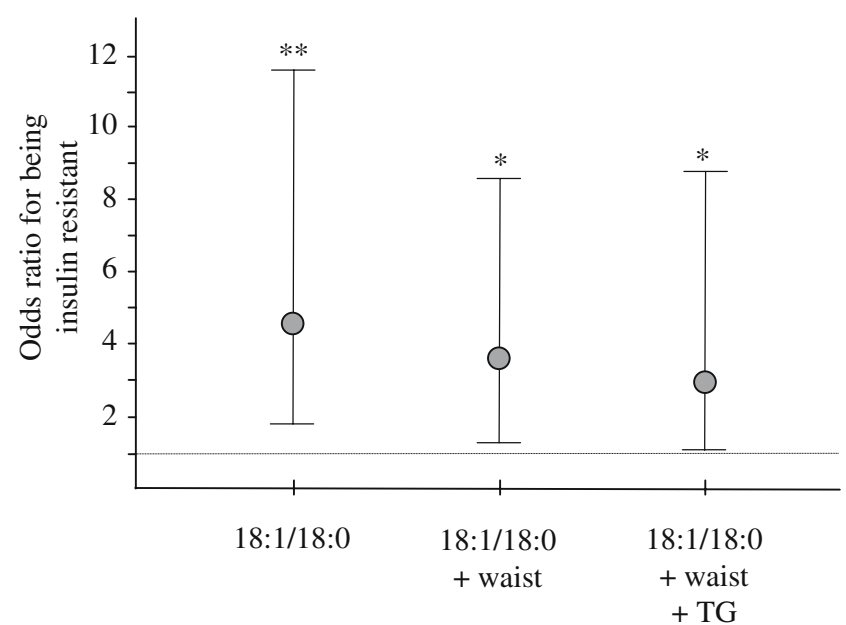

Fig. 2 Odds ratios $(95 \% \mathrm{CI})$ for the effect of the adipose tissue 18:1/ 18:0 ratio on insulin resistance according to logistic regression analysis $(n=289) .{ }^{*} p<0.05,{ }^{*} p<0.01$. Waist, waist circumference; TG, plasma triacylglycerol

\section{Discussion}

Data from this cohort of 62- to 64-year-old men have been reported previously [11-13, 18, 19]. Here, novel information on the expression of desaturase genes in human adipose tissue, relationships to desaturation indexes and association with insulin resistance are presented.

We show that mRNA expression levels of different fatty acid desaturases in adipose tissue are related to each other and that, to some extent, the fatty acid composition of adipose tissue can be used as a biomarker of desaturase gene

Table 3 Univariate and multivariate linear regression analyses for HOMA-2 index

\begin{tabular}{|c|c|c|c|c|}
\hline & \multicolumn{2}{|c|}{ Univariate analysis } & \multicolumn{2}{|c|}{ Multivariate analysis $^{\mathrm{a}}$} \\
\hline & $r$ value & $p$ value & $\beta \pm \mathrm{SE}$ & $p$ value \\
\hline BMI & 0.55 & $<0.0001$ & & \\
\hline Waist circumference & 0.55 & $<0.0001$ & $0.025 \pm 0.003$ & $<0.0001$ \\
\hline Uric acid & 0.29 & $<0.0001$ & & \\
\hline Systolic blood pressure & 0.19 & 0.0012 & & \\
\hline Diastolic blood pressure & 0.24 & $<0.0001$ & & \\
\hline Triacylglycerol & 0.46 & $<0.0001$ & $0.78 \pm 0.14$ & $<0.0001$ \\
\hline HDL-cholesterol & -0.34 & $<0.0001$ & & \\
\hline LDL peak & -0.34 & $<0.0001$ & & \\
\hline C-reactive protein & 0.25 & $<0.0001$ & & \\
\hline Adipose tissue $16: 1 / 16: 0$ & 0.01 & 0.93 & & \\
\hline Adipose tissue $18: 1 / 18: 0$ & 0.27 & $<0.0001$ & $0.46 \pm 0.27$ & 0.086 \\
\hline
\end{tabular}

$n=289$

Prior to analysis, HOMA-2 index, BMI, uric acid, triacylglycerol, CRP and the adipose tissue ratios 16:1/16:0 and 18:1/18:0 were log-transformed

${ }^{a}$ Parsimonious multivariate model (adjusted $R^{2}=0.36$ ). From the variables investigated in univariate analysis, independent predictors of HOMA-2 were identified using a forward stepwise approach (BMI and LDL peak were not included in the model due to strong co-linearity with waist and triacylglycerol, respectively). The final model includes these independent predictors (waist circumference and plasma triacylglycerol) plus the 18:1/18:0 ratio 
expression. The relevant desaturation indexes (i.e. the 16:1/16:0, $18: 1 / 18: 0,20: 4 / 20: 3$ and 18:3/18:2 ratios) reflected the expression of $S C D$, but not $D 5 D$ or $D 6 D$. Individuals with insulin resistance had a higher 18:1/18:0, but not 16:1/16:0 ratio than insulin-sensitive individuals. Indeed, a high 18:1/18:0 ratio was associated with a significantly higher risk of insulin resistance in logistic regression analysis, with the 18:1/18:0 ratio tending to predict insulin resistance in a multiple linear regression model. These results suggest that adipose tissue desaturation indexes of SCD reflect expression of the enzyme in this tissue and that elevated SCD activity within adipose tissue accompanies the development of insulin resistance.

To our knowledge, only one study has previously presented relationships between levels of adipose tissue $S C D$ mRNA and insulin resistance in man. An improvement in insulin sensitivity (by thiazolidinedione treatment) in 24 overweight individuals with type 2 diabetes was concomitant with increased adipose tissue $S C D$ expression, but no direct relationship between changes in $S C D$ expression and enhanced insulin sensitivity was found [20]. That study, however, was small and no data were available to relate $S C D$ expression levels to estimates of SCD activity in adipose tissue. The inherent difficulty of measuring desaturase activities directly has led to the use of fatty acid product/substrate ratios as surrogate estimates of desaturase activities. This approach is supported to some degree by results from animal studies [21-24], but compelling evidence is lacking. Importantly, the absence of human data on the validity of these relationships is of concern, since mice, in contrast to humans, express various isoforms of SCD with different affinities for the precursor fatty acids [4]. In the current study, SCD desaturation indexes (derived from adipose tissue fatty acid composition) were significantly related to the level of adipose tissue $S C D$ mRNA expression. In contrast, no relationships were found between desaturation indexes and the expression of $D 5 D$ or $D 6 D$. This could be explained by the high bioactivity of the products of D5D and D6D, resulting in fatty acid ratios that reflect a range of pathways, rather than just the enzyme activities as such. Thus our expression data indicate that fatty acid ratios can be used to estimate SCD but not D5D or D6D activities in human adipose tissue.

The important role of SCD as a metabolic regulator has been described in various mouse models [4]. SCD-deficient mice are resistant to diet-induced obesity and have increased energy expenditure, reduced lipogenesis and increased insulin sensitivity. The underlying explanation is not clear, but includes enhanced phosphorylation of insulin-receptor substrates and activation of AMP-kinase [25]. Human studies report relationships between estimates of hepatic SCD activity and both plasma triacylglycerol concentrations and obesity $[7,8,26]$. However, these studies have not addressed desaturation activity in adipose tissue, since fatty acid ratios derived from circulating lipids primarily reflect hepatic desaturation activity.

Our results suggest that elevated SCD activity in adipose tissue could contribute to the development of insulin resistance. Indeed, a high adipose tissue 18:1/18:0 ratio was associated with a threefold risk of being insulin resistant, even after adjusting for waist circumference and plasma triacylglycerol. A recent publication identified obesity as being mainly responsible for explaining the association between higher hepatic SCD activity (as estimated from plasma desaturation indexes) and elevated risk of developing the metabolic syndrome [9]. However, the present findings indicate that the relationship between adipose tissue SCD activity and insulin resistance is at least partly independent of obesity (as shown in both logistic and linear regression models). This effect might be mediated through abrogation of insulin signalling pathways and/or AMP-kinase activity, as indicated in animal studies [25]. On the other hand, it is also possible that increases in adipose tissue SCD activity are merely a consequence of the development of insulin resistance. However, thiazolidinedione (insulin-sensitiser) treatment of patients with type 2 diabetes leads to increases in adipose tissue $S C D$ expression, suggesting a beneficial effect of increased adipose tissue SCD activity in these individuals $[20,27]$. One interpretation of these seemingly conflicting data is that the consequences of increased adipose tissue SCD activity depend on the current metabolic status. In insulin-sensitive adipose tissue, there may be a flexible and appropriately regulated balance between up- and downregulation of SCD activity, a balance that shifts according to metabolic demand. However, in insulinresistant adipose tissue an inappropriately prolonged and maintained increase in SCD activity may have detrimental consequences. Indeed, the complexity of the consequences of altering SCD activity was highlighted recently, when SCD deficiency was shown to improve insulin sensitivity in lean mice, but to worsen diabetes in obese littermates [28].

The fact that the 16:1/16:0 ratio, another estimate of SCD activity, did not relate to insulin resistance in this study (as did the 18:1/18:0 ratio) calls for some attention. It is not presently clear which ratio most accurately mirrors SCD activity. The corresponding mouse SCD (namely SCD-1, the enzyme knocked out in SCD-deficient mice) preferentially converts 18:0 to $18: 1$ rather than 16:0 to $16: 1$ [4]. Whether this is the case for human SCD is not clear and both fatty acid ratios have been used in the literature, but not usually concurrently. In contrast to our results, Warensjö et al. [9] identified the circulating 16:1/16:0 ratio, but not 18:1/18:0 ratio, as a predictor of the metabolic syndrome. The authors 
suggested that dietary factors could explain the absent relationship for 18:1/18:0, since the diet is normally high in $18: 1$, which might attenuate the impact of SCD on this specific ratio. Although dietary habits have been shown to influence SCD activity [4], the differing relationships between SCD indexes 16:1/16:0 and 18:1/18:0 and insulin resistance observed in the present study did not appear to be explained by differences in fatty acid intake. Therefore differences in the metabolism of individual fatty acids within adipose tissue may underlie the inconsistent relationships for the two desaturation indexes, but the specific nature of these differences remains unknown.

There are certain limitations to the current study. First, the study is cross-sectional, restricting possibilities of drawing causal conclusions. Second, actual activities of the desaturases were not determined. Instead, mRNA expression levels of desaturases in adipose tissue and the corresponding fatty acid ratios were used as estimates of enzyme activity. Since SCD protein undergoes rapid degradation [29], this could suggest that SCD activity is determined primarily by transcription of the gene and hence by $S C D$ mRNA levels. Finally, the present findings are restricted to SCD in subcutaneous adipose tissue. It is, however, possible that SCD activity in other tissues (such as liver and skeletal muscle) or in other adipose tissue depots has different metabolic consequences. Indeed, a central role has been ascribed to intra-abdominal fat in the development of insulin resistance and differences in SCD activity between subcutaneous and visceral adipose tissue may be important in this context.

In summary, we have shown that the adipose tissue desaturation indexes (fatty acid ratios) of 16:1/16:0 and 18:1/18:0 reflected $S C D$ expression in adipose tissue. In contrast, desaturation indexes for D5D and D6D were not related to their respective gene expression levels. A high SCD desaturation index (18:1/18:0) in adipose tissue increased the risk of being insulin resistant, even after adjusting for waist and plasma triacylglycerol; this index also contributed to a predictive model for insulin resistance. Collectively, these results suggest that derangements in adipose tissue SCD activity might reflect disturbed adipose tissue metabolism, which is closely coupled to the development of insulin resistance.

Acknowledgements This work was supported by the Swedish Research Council (project 15352), the Swedish Diabetes Association, the Novo Nordisk Foundation, the Stockholm County Council, the Swedish Heart and Lung Foundation, The Swedish Nutrition Foundation, the Swedish Council for Working Life and Social Research, a European Foundation for the Study of Diabetes/Lilly Research Fellowship and faculty funds from the Board of Post-Graduate Education of Karolinska Institutet.

Duality of interest The authors declare that there is no duality of interest associated with this manuscript.

\section{References}

1. Food and Agriculture Organization/World Health Organization (2003) Recommendations for preventing type 2 diabetes. In: Diet, nutrition and the prevention of chronic diseases. Report of a joint WHO/FAO expert consultation. WHO technical report series 196. WHO, Geneva, pp 72-80. Available from http://www.who.int/entity/ dietphysicalactivity/publications/trs916/en/gsfao_diabetes.pdf, accessed 26 October 2007

2. Nakamura MT, Nara TY (2004) Structure, function, and dietary regulation of $\Delta 6, \Delta 5$, and $\Delta 9$ desaturases. Annu Rev Nutr 24 : $345-376$

3. Das UN (2005) A defect in the activity of $\Delta 6$ and $\Delta 5$ desaturases may be a factor predisposing to the development of insulin resistance syndrome. Prostaglandins Leukot Essent Fatty Acids 72: 343-350

4. Ntambi JM, Miyazaki M (2004) Regulation of stearoyl-CoA desaturases and role in metabolism. Prog Lipid Res 43:91-104

5. Vessby B (2003) Dietary fat, fatty acid composition in plasma and the metabolic syndrome. Curr Opin Lipidol 14:15-19

6. Ntambi JM, Miyazaki M, Dobrzyn A (2004) Regulation of stearoyl-CoA desaturase expression. Lipids 39:1061-1065

7. Attie AD, Krauss RM, Gray-Keller MP et al (2002) Relationship between stearoyl-CoA desaturase activity and plasma triglycerides in human and mouse hypertriglyceridemia. J Lipid Res 43:1899-1907

8. Shiwaku K, Hashimoto M, Kitajima K et al (2004) Triglyceride levels are ethnic-specifically associated with an index of stearoylCoA desaturase activity and n-3 PUFA levels in Asians. J Lipid Res 45:914-922

9. Warensjo E, Riserus U, Vessby B (2005) Fatty acid composition of serum lipids predicts the development of the metabolic syndrome in men. Diabetologia 48:1999-2005

10. Rosell M, De Faire U, Hellenius ML (2003) Low prevalence of the metabolic syndrome in wine drinkers - is it the alcohol beverage or the lifestyle? Eur J Clin Nutr 57:227-234

11. Sjogren P, Rosell M, Skoglund-Andersson C et al (2004) Milkderived fatty acids are associated with a more favorable LDL particle size distribution in healthy men. J Nutr 134:1729-1735

12. Sjogren P, Basu S, Rosell M et al (2005) Measures of oxidized low-density lipoprotein and oxidative stress are not related and not elevated in otherwise healthy men with the metabolic syndrome. Arterioscler Thromb Vasc Biol 25:2580-2586

13. Gertow K, Rosell M, Sjogren P et al (2006) Fatty acid handling protein expression in adipose tissue, fatty acid composition of adipose tissue and serum, and markers of insulin resistance. Eur J Clin Nutr 60:1406-1413

14. Boberg M, Croon LB, Gustafsson IB, Vessby B (1985) Platelet fatty acid composition in relation to fatty acid composition in plasma and to serum lipoprotein lipids in healthy subjects with special reference to the linoleic acid pathway. Clin Sci (Lond) 68:581-587

15. Wallace TM, Levy JC, Matthews DR (2004) Use and abuse of HOMA modeling. Diabetes Care 27:1487-1495

16. HOMA Calculator, Diabetes Trials Unit, The Oxford Centre for Diabetes, Endocrinology and Metabolism, Oxford, UK. Available from http://www.dtu.ox.ac.uk/homa/index, accessed 26 October 2007

17. Grundy SM, Cleeman JI, Daniels SR et al (2005) Diagnosis and management of the metabolic syndrome: an American Heart Association/National Heart, Lung, and Blood Institute Scientific Statement. Circulation 112:2735-2752

18. Rosell M, Johansson G, Berglund L, Vessby B, de Faire U, Hellenius ML (2004) Associations between the intake of dairy fat and calcium and abdominal obesity. Int J Obes Relat Metab Disord 28:1427-1434 
19. Rosell M, Johansson G, Berglund L, Vessby B, de Faire U, Hellenius ML (2005) The relation between alcohol intake and physical activity and the fatty acids 14:0, 15:0 and 17:0 in serum phospholipids and adipose tissue used as markers for dairy fat intake. Br J Nutr 93:115-121

20. Riserus U, Tan GD, Fielding BA et al (2005) Rosiglitazone increases indexes of stearoyl-CoA desaturase activity in humans: link to insulin sensitization and the role of dominant-negative mutation in peroxisome proliferator-activated receptor-gamma. Diabetes 54:1379-1384

21. Pan DA, Hulbert AJ, Storlien LH (1994) Dietary fats, membrane phospholipids and obesity. J Nutr 124:1555-1565

22. Narce M, Poisson JP, Belleville J, Chanussot B (1988) Timecourse effects of protein malnutrition on hepatic fatty acids $\Delta 6$ and $\Delta 5$ desaturation in the growing rat. $\mathrm{Br} \mathrm{J}$ Nutr 60: 389-402

23. Ulmann L, Poisson JP, Blond JP, Bezard J (1991) Incorporation into liver microsomal lipids of linoleic and stearic acids and of their respective products of $\Delta 6$ and $\Delta 9$ desaturation, $\gamma$-linolenic and oleic acids: effect of age and of blackcurrant seed oil. Biochim Biophys Acta 1086:230-236
24. Wahl HG, Kausch C, Machicao F, Rett K, Stumvoll M, Haring HU (2002) Troglitazone downregulates $\Delta-6$ desaturase gene expression in human skeletal muscle cell cultures. Diabetes 51:1060-1065

25. Dobrzyn A, Ntambi JM (2005) The role of stearoyl-CoA desaturase in the control of metabolism. Prostaglandins Leukot Essent Fatty Acids 73:35-41

26. Warensjo E, Ohrvall M, Vessby B (2006) Fatty acid composition and estimated desaturase activities are associated with obesity and lifestyle variables in men and women. Nutr Metab Cardiovasc Dis $16: 128-136$

27. Kolak M, Yki-Jarvinen H, Kannisto K et al (2007) Effects of chronic rosiglitazone therapy on gene expression in human adipose tissue in vivo in patients with type 2 diabetes. J Clin Endocrinol Metab 92:720-724

28. Flowers JB, Rabaglia ME, Schueler KL et al (2007) Loss of stearoylCoA desaturase-1 improves insulin sensitivity in lean mice but worsens diabetes in leptin-deficient obese mice. Diabetes 56:1228-1239

29. Heinemann FS, Ozols J (2003) Stearoyl-CoA desaturase, a shortlived protein of endoplasmic reticulum with multiple control mechanisms. Prostaglandins Leukot Essent Fatty Acids 68:123-133 